\title{
INTEGRIDAD ELECTORAL, INTERÉS EN LA POLÍTICA Y SATISFACGIÓN CON LA DEMOCRACIA EN MÉXICO
}

\author{
ELECTORAL INTEGRITY, INTEREST IN POLITICS, AND \\ SATISFACTION WITH DEMOCRACY IN MEXICO
}

\section{INTÉGRITÉ ÉLECTORALE, INTÉRÊT POLITIQUE ET SATISFACTION À L'ÉGARD DE LA DÉMOCRATIE AU MEXIQUE}

\author{
Alejandro Monsiváis Carrillo ${ }^{1}$ \\ El Colegio de la Frontera Norte \\ amonsi@colef.mx
}

\begin{abstract}
RESUMEN: La opinión pública acerca de la integridad de los procesos electorales es tan importante para la satisfacción con la democracia como la calidad de las elecciones. Una cuestión poco explorada, sin embargo, es si la integridad electoral tiene la misma importancia para todos los ciudadanos. El argumento de este estudio es que quienes más se interesan en los asuntos públicos también valoran más la calidad de las elecciones. Con base en un análisis del caso de México, los resultados muestran que la percepción de manipulación electoral disminuye la satisfacción con la democracia, más aún entre los ciudadanos más interesados en la política.
\end{abstract}

Palabras clave: satisfacción con la democracia; percepción de integridad electoral; percepción de manipulación electoral; interés en la política.

Aвstract: Citizen perceptions of electoral integrity play an influential role in their assessment of democracy. This research argues that citizens' perceptions of electoral integrity and malpractice are further conditioned by their level of interest in politics. If elections are perceived as free and fair, those who are more interested in politics are expected to be more satisfied with democracy. Focusing on the Mexican case, the analysis shows that interest in politics

${ }^{1} \mathrm{El}$ autor expresa su agradecimiento a los revisores anónimos de este artículo por sus valiosas observaciones y recomendaciones. 
conditions the relationship between perceptions of electoral malpractice and satisfaction with democracy. Citizens who are more interested in politics are less satisfied with democracy when they perceive evidence of fraud or electoral manipulation.

Keywords: satisfaction with democracy; perception of electoral integrity; perception of electoral malpractice; political interest; Mexico.

\section{Traducción de Fionn Petch, CM Idiomas}

Résumé : L'opinion publique sur l'intégrité des processus électoraux est aussi importante pour la satisfaction vis-à-vis de la démocratie que la qualité des élections. Une question peu explorée, cependant, est de savoir si l'intégrité électorale est d'égale importance pour tous les citoyens. La thèse de cette étude est que ceux qui s'intéressent le plus aux affaires publiques apprécient également davantage la qualité des élections. Sur la base d'une analyse du cas du Mexique, les résultats montrent que la perception d'une manipulation électorale diminue la satisfaction à l'égard de la démocratie, d'autant plus chez les citoyens les plus intéressés par la politique.

Mots-cléf: satisfaction à l'égard de la démocratie ; perception de

l'intégrité électorale; perception de la manipulation électorale; intérêt envers la politique.

Traducción de Rafael Segovia, CM Idiomas

Fecha de recepción: enero de 2021.

Fecha de aceptación: mayo de 2021. 


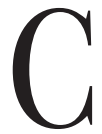

elebrar elecciones en condiciones de integridad es una circunstancia necesaria de la democracia. La integridad de las elecciones implica que las leyes y normas garantizan una competencia inclusiva y equitativa, que los procesos son transparentes e imparciales durante el ciclo electoral, y que existe certeza acerca de la limpieza de los resultados. ${ }^{2}$ La integridad electoral supone, también, que las prácticas fraudulentas o de manipulación han sido minimizadas o, idealmente, erradicadas. Sin un mínimo de integridad, las elecciones podrán contribuir a que los gobernantes se mantengan en el poder pretendiendo contar con legitimidad popular, pero difícilmente podrán ser consideradas elecciones propias de una democracia. ${ }^{3}$

Tan importante como la calidad de las elecciones es la creencia ciudadana de que los procesos electorales son justos e imparciales. La percepción de integridad electoral influye positivamente en la satisfacción de los ciudadanos con la democracia, de la misma forma que la percepción de manipulación electoral impacta de forma negativa en la evaluación del régimen. ${ }^{4}$ La satisfacción con la democracia, al igual

2 Pippa Norris, Why Electoral Integrity Matters, Cambridge, University Press, 2014, p. 21; Carolien van Ham, "Getting elections right? Measuring electoral integrity”, Democratization 22, núm. 4 (2015); Irma Méndez de Hoyos, Ferrán Martínez i Coma y Nicolás Loza, "Introducción", en Irma Méndez, Nicolás Loza y Ferrán Martínez (eds.), Integridad electoral: México en perspectiva global, México, Flacso-México, 2019. Contribuciones originales a esta agenda de investigación, al igual que bases de datos y otra información valiosa, se encuentra en: https:/ / www.electoralintegrityproject.com/

${ }^{3}$ Andreas Schedler, The Politics of Uncertainty: Sustaining and Subverting Electoral Authoritarianism, Oxford, University Press, 2013.

${ }^{4}$ Pippa Norris, "Do Perceptions of Electoral Malpractice Undermine Democratic Satisfaction? The US in Comparative Perspective", International Political Science Review 40, núm. 1 (2019); Jessica Fortin-Rittberger, Philipp Harfst y Sarah C. Dingler, "The Costs of Electoral Fraud: Establishing the Link Between Electoral Integrity, Winning an Election, and Satisfaction with Democracy", Journal of Elections, Public Opinion and Parties 27, núm. 3 (2017); Ian McAllister y Stephen White, "Electoral Integrity and Support for Democracy in Belarus, Russia, and Ukraine", Journal of Elections, Public Opinion and Parties 25, núm. 1 (2015). 
que otras expresiones de confianza en las instituciones representativas o en el sistema político, se considera un factor que contribuye a la estabilidad y profundización de la democracia. ${ }^{5}$ Cuando predomina la desconfianza en la calidad de los procesos electorales, la insatisfacción democrática puede, a la vez, ocasionar inestabilidad o favorecer su declive.

Un aspecto todavía poco estudiado en la literatura, sin embargo, es la importancia relativa que los ciudadanos pueden darle a la integridad electoral. Si bien las elecciones libres y limpias tienden a fortalecer la satisfacción con la democracia, es posible que algunos ciudadanos sean más sensibles que otros a la calidad de las elecciones. Un factor que puede marcar una diferencia en ese sentido es el interés en la política, una actitud que refleja una valoración positiva del involucramiento en cierto campo de actividades ${ }^{6}$-la "política”, en este caso-, y que desempeña un rol importante como predictor de la participación ciudadana. ${ }^{7}$ En ese sentido, los ciudadanos más interesados en la política pueden verse más afectados por el grado en que "está pareja la cancha" o la competencia electoral se desarrolla en condiciones de equidad y libertad.

El argumento que se desarrolla en este estudio es que el interés en la política desempeña un rol condicional en la relación entre las percepciones de integridad o manipulación electoral en la evaluación de la democracia. Esto signi-

5 Larry Diamond, Developing Democracy: Toward Consolitadion, Baltimore, The John Hopkins University Press, 1999, pp. 168-169; John A. Booth y Mitchell A. Seligson, The Legitimacy Puzzle in Latin America: Political Support and Democracy in Eight Nations, Cambridge, Nueva York, Cambridge University Press, 2009.

${ }^{6}$ Joshua Robison, "The Social Rewards of Engagement: Appealing to Social Motivations to Stimulate Political Interest at High and Low Levels of External Efficacy", Political Studies 65, núm. 1 (2017).

7 Por ejemplo, Sidney Verba, Kay Lehman Schlozman y Henry E Brady, Voice and Equality: Civic Voluntarism in American Politics, Harvard, University Press, 1995. 
fica que la influencia positiva o negativa que ejerzan esas percepciones tendrá un impacto diferente, dependiendo de qué tanto se interese la ciudadanía en los asuntos públicos. La expectativa es que las personas que más se interesen por la política reaccionen más positivamente ante la integridad de las elecciones y de forma más negativa ante la evidencia de malas prácticas.

Para evaluar este planteamiento, el análisis se enfoca en el caso de México. En este país, el establecimiento de una democracia electoral fue resultado de la creación de instituciones de administración y justicia electoral autónomas, que garantizaran un piso mínimo para celebrar elecciones libres y limpias. ${ }^{8}$ A lo largo de más de dos décadas, las instituciones de gobernanza electoral han acumulado aprendizajes y desarrollado capacidades para organizar elecciones cumpliendo con estándares internacionales. A pesar de todo, la ciudadanía tiene poca confianza en la calidad de los procesos electorales. Como lo muestran diversos estudios, la confianza de los mexicanos en la limpieza de las elecciones y en la imparcialidad de las autoridades electorales ha disminuido sistemáticamente en los últimos años. ${ }^{9}$ En buena medida, esto tiene su origen en los conflictos entre los candidatos y sus partidos políticos en torno a los resultados de las elec-

${ }^{8}$ Para un panorama general de ese proceso, de entre una amplia variedad de fuentes igualmente relevantes, véase: José Woldenberg, Historia mínima de la transición democrática en México, México, El Colegio de Mexico, 2012.

${ }^{9}$ Cfr. Yuritzi Mendizábal y Alejandro Moreno, "La confianza electoral: el IFE y los partidos políticos", en La confianza en las instituciones, Alejandro Moreno (ed.), México, Centro de Estudios Sociales y de Opinión Pública-Cámara de Diputados, 2010; Reynaldo Yunuen Ortega y María Fernanda Somuano, Confianza y cambio político en México: contiendas electorales y el IFE, México, El Colegio de México, 2015, p. 60; Willibald Sonnleitner, La fábrica de la (des)confianza ciudadana: las percepciones cambiantes de la integridad electoral en México, México, CEsop-Cámara de Diputados, 2016; Germán Pérez-Verduzco, "Confianza en el Instituto Nacional Electoral mexicano: una perspectiva comparada”, Reflexión Política 22, núm. 45 (2020). 
ciones. ${ }^{10}$ Con todo, el análisis de las consecuencias de la desconfianza de los mexicanos en los procesos electorales ha recibido poca atención. Aunque diversas investigaciones han documentado el aumento del descontento político, ${ }^{11}$ se ha investigado poco el impacto de la percepción de integridad electoral en la evaluación del desempeño de la democracia.

Este estudio analiza las consecuencias de la percepción de integridad electoral en la evaluación de la democracia en México, presentando evidencia de que el interés de la ciudadanía en los asuntos públicos es un factor que condiciona su opinión acerca de la calidad de las elecciones. El análisis muestra que, en este país, prevalece la opinión de que persisten las malas prácticas electorales antes que la creencia de que las elecciones son libres y limpias. La percepción de manipulación electoral afecta negativamente la satisfacción con la democracia. De hecho, los ciudadanos que más se interesan en la política son quienes se encuentran menos satisfechos cuando perciben que las prácticas de manipulación electoral están generalizadas.

${ }^{10}$ Sonnleitner, op. cit.; Antonio Ugues Jr., "Citizens' Views on Electoral Governance in Mexico", Journal of Elections, Public Opinion and Parties 20, núm. 4 (2010); Antonio Ugues Jr. y D. Xavier Medina Vidal, "Public evaluations of electoral institutions in Mexico: An analysis of the IFE and TRIFE in the 2006 and 2012 elections", Electoral Studies 40 (2015); Antonio Ugues Jr., "Public Perceptions of Clean Elections in Mexico: An Analysis of the 2000, 2006, and 2012 Elections", Journal of Politics in Latin America 10, núm. 2 (2018).

${ }^{11}$ Alejandro Moreno, "Citizen's Values and Beliefs toward Politics: Is Democracy Growing Attitudinal Roots?", en Andrew Seele y Jacqueline Peschard, (eds.), Mexico's Democratic Challenges: Politics, Government, and Society, Washington, Standford, Woodrow Wilson Center Press, Stanford University Press, 2010; Alejandro Moreno y Shannon K. O’Neil, "El malestar democrático en México", en Gustavo Meixueiro y Alejandro Moreno (eds.), El comportamiento electoral mexicano en las elecciones de 2012, México, CESop-Cámara de Diputados (LXII Legislatura), 2014; Alejandro Monsiváis Carrillo, "La insatisfacción con la democracia en México (20142017)", Perfiles Latinoamericanos 27, núm. 53 (2019); María Fernanda Somuano, "Los determinantes de la legitimidad gubernamental: el sexenio de Enrique Peña Nieto", Foro Internacional 60, núm. 2 (2020). 
El texto se organiza de la siguiente forma: la primera sección introduce el tema de la satisfacción con la democracia. La segunda sección discute el rol de la integridad electoral en la evaluación de los ciudadanos del desempeño de la democracia en la que viven. En esta parte se argumenta que el interés en la política puede condicionar la relación entre las percepciones de integridad en las elecciones y la evaluación del régimen. Posteriormente se describen los componentes del diseño metodológico. Las secciones siguientes describen la información empírica y exponen los resultados del análisis. La última parte discute los hallazgos y ofrece un balance general de la problemática y los resultados obtenidos.

SATISFACCIÓN CON LA DEMOCRACIA E INTEGRIDAD ELECTORAL

La satisfacción con el desempeño de la democracia es una pieza clave del vínculo entre la ciudadanía y el sistema político. De forma similar a la confianza en las instituciones políticas, la satisfacción con la democracia es una actitud evaluativa del funcionamiento de sistemas políticos concretos. ${ }^{12}$ A diferencia de la preferencia por la democracia, que concierne al valor que la ciudadanía le otorga a este régimen, la satisfacción democrática sólo juzga su desempeño. Puede darse el caso de que el apoyo hacia la democracia sea amplio, pero también que exista un alto grado de insatisfacción con su operación. De la misma forma, algunas personas pueden declararse escépticas acerca de la legitimidad de la democracia, aunque estén satisfechas con el desempeño de la democracia en la que viven.

12 Jonas Linde y Joakim Ekman, "Satisfaction with Democracy: A Note on a Frequently Used Indicator in Comparative Politics", European Journal of Political Research 42, núm. 3 (2003); Tom W.G. van der Meer y Sonja Zmerli, "The Deeple Rooted Concern with Political Trust", en Tom W.G. van der Meer y Sonja Zmerli (eds.), Handbook on Political Trust, Reino Unidos, Edward Elgar Publishing, 2017. 
De acuerdo con la literatura especializada, uno de los principales factores asociados a la satisfacción con la democracia es el sentimiento de representación de los votantes. La satisfacción entre personas que votaron a favor de los candidatos o partidos que están en el poder tiende a ser más alta que entre las personas que votaron por candidatos o partidos que perdieron las elecciones. ${ }^{13}$ De igual forma, un número creciente de estudios revela que la satisfacción con el régimen, depende de qué tanto la ciudadanía tenga una comprensión correcta de la democracia. Entre más informada sea su comprensión del régimen y más democrático sea el sistema en el que viven, es más probable que su evaluación de la calidad de la democracia sea positiva. ${ }^{14}$ Por otra parte, la literatura comparada muestra que los votantes premian el desempeño del sistema político no sólo por los beneficios que reciben en cuanto a desarrollo económico o bienestar social. Los votantes valoran, igualmente, la calidad del gobierno y la imparcialidad de los procedimientos. ${ }^{15}$ Cuando los procesos son justos e imparciales, la satisfacción con la democracia persiste, aunque los resultados sean poco convincentes.

La centralidad que tiene la calidad de los procedimientos en la evaluación del régimen sugiere que la integridad de las

13 Véase, inter alia: Christopher J. Anderson et al., Loser's Consent: Elections and Democratic Legitimacy, Oxford, University Press, 2005.

14 Youngho Cho, "To Know Democracy Is to Love It:A Cross-National Analysis of Democratic Understanding and Political Support for Democracy”, Political Research Quarterly 67, núm. 3 (2014); Carsten Wegscheider y Toralf Stark, "What Drives Citizens' Evaluation of Democratic Performance? The Interaction of Citizens' Democratic Knowledge and Institutional Level of Democracy", Zeitschrift für Vergleichende Politikwissenschaft 14, núm. 4 (2020).

15 Pippa Norris, Democratic Deficit: Critical Citizens Revisited, Cambridge, University Press, 2011; Jonas Linde, "Why Feed the Hand that Bites You? Perceptions of Procedural Fairness and System Support in Post-Communist Democracies”, European Journal of Political Research 51, núm. 3 (2012); Pedro C. Magalhães, "Economic Evaluations, Procedural Fairness, and Satisfaction with Democracy", Political Research Quarterly 69, núm. 3 (2016). 
elecciones desempeña un rol clave en la satisfacción democrática. La integridad de las elecciones atañe a las condiciones en las que se realizan los comicios, independientemente de qué candidaturas resulten electas. Desde un punto de vista procedimental, lo que importa es que las elecciones se conduzcan de forma imparcial, para que los votantes ejerzan en libertad sus derechos y los competidores encuentren un piso parejo.

\section{INTEGRIDAD ELECTORAL, INTERÉS EN LA POLÍTICA Y SATISFACCIÓN DEMOCRÁTICA}

La idea de que las elecciones democráticas deben ser libres y limpias no tendría que ser un tópico demasiado controvertido. Para diversos especialistas, la controversia radica en cómo conceptualizar y, sobre todo, como observar empíricamente los atributos de las elecciones. ${ }^{16}$ Si bien existe coincidencia en que éstas deben ser de buena calidad, íntegras, competitivas, o libres de fraude e imparciales, también hay coincidencia en los desafíos metodológicos que persisten en el estudio de los procesos electorales.

En este debate, el concepto de integridad electoral ha contribuido a clarificar los estándares que deben cumplir las elecciones democráticas. La integridad electoral es el cumplimiento de las normas reconocidas en tratados y convenciones internacionales para garantizar que las elecciones sean libres, competitivas e imparciales. ${ }^{17}$ El cumplimiento de tales normas debe ser universal -lo mismo para democracias establecidas que para todo régimen en el que se lleven a cabo procesos electorales-. Esas normas han de garantizarse du-

${ }^{16}$ Ham, art. cit.; Robert A. Pastor, art. cit., p. 6; Sylvia Bishop y Anke Hoeffler, "Free and fair elections: A new database", Journal of Peace Research 53, núm. 4 (2016).

${ }^{17}$ La definición que se presenta aquí se desarrolla en: Norris, Why Electoral Integrity Matters, op. cit., p. 21. Véase, también, la nota 2 de este trabajo. 
rante todo lo largo del ciclo electoral, desde el diseño y aprobación de leyes, la profesionalización del servicio electoral, el registro de votantes y partidos políticos, la certificación de candidatos, entre otros, hasta la logística del día de la elección, incluyendo los procesos de conteo, emisión de resultados y resolución de controversias, y demás. El concepto de integridad electoral también evalúa la presencia de manipulación y malas prácticas, bajo la premisa de que las incidencias de este tipo deben prevenirse o estar ausentes de los procesos electorales. Se trata de prácticas como la compra y coacción del voto, las leyes que limitan la participación de partidos de oposición o prohíben la postulación de candidaturas legítimas, la regulación precaria o ineficaz de las campanas electorales, la parcialidad de los medios de comunicación -a favor del partido gobernante, por ejemplo-, o inclusive los actos de violencia durante la campaña, la jornada electoral o luego de publicarse los resultados.

La integridad de las elecciones reviste una especial importancia para la legitimidad de la democracia. ${ }^{18}$ Un número creciente de estudios demuestra, de hecho, que tanto la integridad de las elecciones como la evidencia de fraude o manipulación electoral son factores determinantes de las actitudes hacia las instituciones políticas o la democracia. Los estudios muestran que tanto las malas prácticas como la integridad de facto de los procesos electorales importan en la evaluación que hace la ciudadanía de la democracia, la imparcialidad de las elecciones o las instituciones políticas. ${ }^{19}$ Del mismo modo, se ha encontrado que, conforme la

${ }^{18}$ J. Elklit y A. Reynolds, "The Impact of Election Administration on the Legitimacy of Emerging Democracies: A New Comparative Politics Research Agenda", Commonwealth Ė Comparative Politics 40, núm. 2 (2002).

${ }^{19}$ Sarah Birch, "Electoral institutions and popular confidence in electoral processes: A cross-national analysis", Electoral Studies 27, núm. 2 (2008); Marlene Mauk, "Electoral integrity matters: how electoral process conditions the relationship between political losing and political trust", Quality E Quantity (2020); Nicholas Kerr y Anna Lührmann, "Public trust in manipulated elections: The role of election administration 
ciudadanía percibe que las elecciones se conducen con imparcialidad, profesionalismo y efectividad, su evaluación del desempeño del sistema es más favorable -y a la inversa, la percepción de malas prácticas electorales provoca desconfianza e insatisfacción políticas. ${ }^{20}$

$\mathrm{Al}$ investigar las consecuencias de las percepciones de integridad electoral en la evaluación de la democracia, sin embargo, se ha explorado poco el rol que desempeña el interés en la política como un factor condicionante de esa relación. Se trata de una actitud que refleja la opinión de las personas acerca del valor de involucrarse en temas políticos; como lo ha mostrado Robinson, ${ }^{21}$ el interés en la política refleja la relevancia que las personas le atribuyen a una actividad o dominio de actividades -como la política o los asuntos públicos-, y una consecuente disposición a involucrarse en estas actividades. En ese sentido, el interés en la política es un indicador clave de la sofisticación política de los individuos y un rasgo personal que, a pesar de fluctuaciones coyunturales, persiste a lo largo del tiempo, ${ }^{22}$ sobre todo en la medida en que implica una actitud que valora en mayor o menor medida el involucramiento en temas

and media freedom", Electoral Studies 50 (2017); Shaun Bowler et al., "Election administration and perceptions of fair elections", Electoral Studies 38 (1 de junio de 2015), pp. 1-9, https://doi.org/10.1016/j.electstud. 2015.01.004

${ }^{20}$ Fortin-Rittberger, Harfst y Dingler, "The Costs of Electoral Fraud: Establishing the Link Between Electoral Integrity, Winning an Election, and Satisfaction with Democracy", art. cit.; Norris, "Do Perceptions...", art. cit.; McAllister y White, "Electoral Integrity and Support for Democracy in Belarus, Russia, and Ukraine”, art. cit.; Holly Ann Garnett, "On the front lines of democracy: perceptions of electoral officials and democratic elections", Democratization 26, núm. 8 (2019).

${ }^{21}$ Robison, "The Social Rewards...", art. cit, pp. 25-26.

22 Robert C. Luskin, "Explaining political sophistication”, Political Behavior 12, núm. 4 (1990); Markus Prior, "You’ve Either Got It or You Don't? The Stability of Political Interest over the Life Cycle", The Journal of Politics 72, núm. 3 (2010). 
políticos, es un predictor sólido de diversas formas de participación cívica y electoral. ${ }^{23}$

Asimismo, el interés en la política puede explicar que algunas personas reaccionen de forma más positiva o negativa ante la calidad de las elecciones. En una democracia, una persona interesada en los asuntos públicos tendrá mayor motivación para informarse acerca de los temas que le resulten prioritarios. Durante las elecciones, es probable que también tenga mayor disposición a estar al tanto de las campañas electorales, las candidaturas y sus propuestas. En ese mismo sentido, al verse más involucrada en el proceso electoral, es probable que esa persona reaccione con mayor satisfacción ante la evidencia de que las elecciones se realizan en condiciones de imparcialidad y profesionalismo. En cambio, si el proceso se ve distorsionado por malas prácticas o intentos de fraude, su juicio acerca del funcionamiento de la democracia reflejará el descontento causado por la manipulación electoral.

En concreto, la primera expectativa empírica es que la percepción de que las elecciones se conducen con integridad tenga efecto positivo en la evaluación del régimen. A saber, si la ciudadanía cree que los votos se cuentan limpiamente, es más probable que tenga una impresión positiva de la democracia. Lo mismo si los votantes perciben que los funcionarios electorales se comportan con profesionalismo e imparcialidad, o los medios de comunicación ofrecen una cobertura equilibrada de las campañas electorales, entre otros posibles indicadores de integridad. Por ende, la primera hipótesis de este estudio es la siguiente:

H1: La percepción de que las elecciones se conducen con integridad producirá un efecto positivo en la satisfacción con la democracia.

${ }^{23}$ Verba, Schlozman y Brady, op. cit.; Lawrence R. Jacobs, Fay Lomax Cook y Michael X. Delli Carpini, Talking Together: Public Deliberation and Political Participation in America, Chicago, University of Chicago Press, 2009. 
La influencia de las percepciones de integridad en la satisfacción con la democracia, sin embargo, debe ser independiente del efecto que pueden tener las experiencias con prácticas fraudulentas o de manipulación. La ciudadanía puede creer en la integridad del proceso, pero también estar al tanto de actividades fraudulentas o que distorsionan la imparcialidad de la contienda. Por ejemplo, ciertos partidos o movimientos de oposición pueden tener prohibido designar candidatos que compitan por los puestos de elección popular. Asimismo, pueden persistir prácticas de compara y coacción del voto, o la violencia política puede afectar el transcurso de la jornada. Así, la evidencia de malas prácticas, dirigidas a distorsionar la imparcialidad del proceso, pueden motivar a los ciudadanos a evaluar negativamente el desempeño de la democracia. De aquí se desprende la segunda hipótesis:

H2: La percepción de manipulación electoral tendrá un efecto negativo en la satisfacción con la democracia.

El argumento central de este estudio, sin embargo, es que el interés en la política modifica la relación entre las percepciones de integridad o manipulación electoral y la satisfacción con la democracia. El supuesto de base es que la importancia que las personas le confieran a la calidad de las elecciones dependerá de qué tan interesadas estén en los asuntos públicos. Quienes más se interesen en la política se verán más impactados por la forma en que se realizan los procesos electorales. Como lo establecen las hipótesis descritas arriba, es de esperar que todos los ciudadanos se sientan más satisfechos con la democracia cuando creen que las elecciones son libres y limpias, o que juzguen negativamente al sistema si perciben un alto grado de manipulación electoral. No obstante, algunos ciudadanos reaccionarán expresando mayor satisfacción (o insatisfacción), dependiendo de qué tan interesados estén en la vida pública. Las hipótesis que se derivan de este planteamiento son las siguientes: 
H3: La relación entre la percepción de integridad electoral y la satisfacción con la democracia está condicionada positivamente por el interés en la política.

H4: La relación entre la percepción de manipulación electoral y la satisfacción con la democracia está condicionada negativamente por el interés en la política.

A saber, la tercera hipótesis postula que, si la percepción de integridad electoral influye positivamente en la satisfacción con la democracia, la satisfacción con el desempeño del régimen será de mayor magnitud entre los ciudadanos que se muestren más interesados en la política. Para quienes se interesan en la política, constatar que las elecciones son libres y limpias puede tener mayor valor que para quienes tienen poco interés. De forma semejante, la cuarta hipótesis predice que el interés en la política modificará en un sentido negativo la relación entre la percepción de manipulación electoral y la satisfacción democrática. Entre los ciudadanos que perciben un nivel específico de malas prácticas electorales, la satisfacción con la democracia será menor para aquellos que estén más interesados en la política.

\section{DisEÑO DE LA INVESTIGACIÓN}

\section{Datos}

Los datos provienen de la ronda 7 de la Encuesta Mundial de Valores (World Value Survey-7 [WVS-7]) en México, ${ }^{24}$ levantada entre los meses de enero y mayo de 2018. El levantamiento precede a las elecciones celebradas en julio de ese

${ }^{24}$ Christian Haerpfer et al., "World Values Survey: Round SevenCountry-Pooled Datafile", Madrid y Viena, JD Systems Institute y wvsA Secretariat, 2020. Los datos están disponibles en: www.wordlvaluesurvey.org 
mismo año, por lo que no pueden considerarse que esos comicios sean el antecedente inmediato de los resultados del análisis. Esta encuesta es representativa a nivel nacional de la población adulta en edad de votar (18 años o más). La muestra consta de 1741 entrevistas realizadas cara a cara en hogares en 496 secciones electorales en las 32 entidades federativas en México. El diseño muestral es probabilístico y multietápico a partir de una lista de 68364 secciones electorales donde se ubicaba el $100 \%$ de los 86 millones de electores registrados por el Instituto Nacional Electoral.

La ventaja que ofrece la wvs-7 es que contiene una batería de reactivos cuyo objetivo explícito es medir la percepción ciudadana sobre la integridad de las elecciones, al igual que un indicador idóneo para analizar la satisfacción con la democracia. ${ }^{25}$

\section{Satisfacción con la democracia}

La evaluación del funcionamiento de la democracia habitualmente depende de reactivos que preguntan por el nivel de satisfacción con el sistema. La encuesta wvs-7 México contiene un reactivo que mide el nivel de democracia que los ciudadanos le atribuyen al sistema político, a saber: "¿Qué tan democrático es el gobierno de México hoy en día?”. Este reactivo está medido en una escala del 1 al 10, donde 1 representa el valor mínimo y 10 el máximo. Esta variable tiene la ventaja de estar medida en una escala continua, a diferencia de los indicadores más comunes de satisfacción con la democracia, que son variables ordinales de cuatro niveles. Por otra parte, la encuesta wvs-7 pregunta expresamente por el nivel

${ }^{25}$ La Encuesta Nacional de Cultura Cívica 2020, resultado de la colaboración entre el Instituto Nacional de Estadística y Geografía (Inegi) y el Instituto Nacional Electoral (INE), también contiene una batería que mide percepciones de integridad y manipulación electoral. Al momento de realizar este estudio, los datos de esas variables aún no habían sido abiertos al público. 
democrático del sistema, lo cual delimita mejor el objeto político que es evaluado por la ciudadanía.

La información estadística descriptiva de todas las variables empleadas en el análisis se muestra en el cuadro 1 del apéndice. Una descripción contextualizada de estos datos se presenta en la siguiente sección, antes de proceder a la exposición de los resultados.

\section{Variables independientes}

Para medir los conceptos de percepción de integridad electoral y percepción de manipulación electoral, se replicó la estrategia utilizada por Pippa Norris. ${ }^{26}$ Esta estrategia, además de contemplar la validación de los índices empíricos de los dos conceptos de interés, permite hacer comparable el análisis con otros estudios que utilicen la misma batería de reactivos. El cuadro A2 del apéndice presenta información desagregada de los ítems que componen los índices de percepción de integridad y manipulación electoral.

Así, la percepción de integridad electoral se mide agregando cinco ítems que preguntan por la frecuencia con la que se observan los siguientes aspectos: "Los votos se cuentan limpiamente"; "Los periodistas hacen una cobertura imparcial de las elecciones"; "Las autoridades electorales son justas"; "Los votantes tienes opciones reales para elegir"; "Las mujeres tienen igualdad de oportunidades para ser candidatas a puestos de elección popular". El índice resultante tiene una confiabilidad de 0.603 (alpha de Cronbach). A su vez, la percepción de manipulación electoral tiene un alpha de Cronbach igual a 0.650 y se compone de los siguientes reactivos: "Se prohíbe competir a candidatos de la oposición"; "Los noticieros de televisión favorecen al partido gobernante"; "Hay compra de votos"; "Los ricos compran las elecciones" y "Se amenaza a los votantes con violencia en las urnas”.

${ }^{26}$ Norris, "Do Perceptions...", art. cit. 
Por otra parte, la variable que desempeña un rol condicional en las hipótesis es el interés en la política. Ésta se mide con una variable ordinal de cuatro categorías. Las opciones de respuesta van desde "nada" hasta "mucho" interés.

\section{Variables de control}

Para someter a prueba las hipótesis es preciso evitar que las estimaciones se vean confundidas por el efecto de alguna variable relevante omitida del análisis. Con tal finalidad, en primer lugar, se introduce una variable que mide importancia de las elecciones. Ésta permite controlar la relevancia que tiene para las personas tener elecciones limpias en un rango de "nada importante" a "muy importante". En segundo lugar, se mide el grado de satisfacción económica de los individuos con la siguiente pregunta: “¿Qué tan satisfecho está con la situación económica de su hogar”. La satisfacción con el desempeño de la economía es considerada, por lo regular, un factor clave en la evaluación del desempeño del sistema político. En tercera instancia, se controla la comprensión de la democracia. Esta variable se obtiene, por un lado, sumando reactivos que identifican atributos de una democracia electoral y, por el otro, restando reactivos que pueden atribuirse a la democracia pero que, en realidad, son atributos de regímenes autoritarios.

Por otro lado, las afinidades políticas de los votantes son consideradas predictores robustos del apoyo político y la satisfacción con la democracia. Se sabe que los votantes "ganadores" -los que votaron por el presidente o el partido gobernante- tienden a ser más generosos en su evaluación del desempeño del sistema político que los "perdedores". Aunque la wvs-7-México no contempla reactivos que permitan identificar a los votantes "ganadores" y a los "perdedores", sí contiene preguntas que captan las identidades partidistas y no-partidistas del electorado. De esta forma, se construyó un indicador de identificación partidista que inclu- 
ye a los ciudadanos que no se identifican con ningún partido o que son antipartidos. En el análisis se toma como referencia a los votantes identificados con el Partido Revolucionario Institucional (PRI), dado que el presidente del país en el momento en que se levantó la encuesta, Enrique Peña Nieto, es miembro de ese partido.

Otro grupo de variables de control incluye indicadores de ideología, consumo informativo y participación en organizaciones sociales. La ideología de la persona entrevistada se mide con base en la posición que adopta en una escala que va de "izquierda" (1) a derecha (10). El consumo informativo es un índice aditivo que reúne a las fuentes y la frecuencia con la que se obtiene información de actualidad. La membresía en organizaciones sociales registra la pertenencia a alguna modalidad de organización social, recreativa o cívica, también mediante un índice aditivo.

Por último, se incluyen las variables sociodemográficas. La variable sexo tiene dos categorías: mujeres (52.2\%) y hombres $(47.8 \%)$. La edad tiene un rango que va de 18 a 90 años, con un promedio de 42 años (usando datos ponderados). En tercer lugar, está el nivel educativo. Esta variable abarca ocho niveles. La cuarta variable es el nivel de ingreso del hogar. En la wvs-7 se pide a los entrevistados que vean una tarjeta en la cual hay una escala con distintos niveles de ingreso del hogar e indiquen en cuál grupo se ubican sus ingresos. De esta forma, las personas entrevistadas no proporcionan a los encuestadores una cantidad monetaria sino un dígito de una escala que va de 0 a 10. Finalmente, se incorpora en los modelos un identificador de residencia (69\%) urbana o ru$\operatorname{ral}(31 \%)$.

\section{Método}

El análisis de los datos se realiza mediante regresiones lineales que estiman los errores estándar a través del método de series linearizadas de Taylor. Este método ajusta las estima- 
ciones tomando en cuenta la estructura compleja del diseño muestral de la encuesta. Si no se considera el diseño de la muestra, los errores estándar pueden subestimarse, produciendo resultados que parecen ser estadísticamente significativos, aún cuando no lo sean. Las variables utilizadas son: la estratificación muestral (N_REGION_ISORegionISO31662) y las unidades primarias de muestreo (I_PSUPrimarySam plingUnitID), ponderando las observaciones por la variable que corrige las estimaciones con base en la distribución de sexo, edad y educación de la población (W_WEIGHTWeight).

\section{ANÁlisis DESCRIPTIVO}

En México, la evaluación del desempeño de la democracia por parte de la ciudadanía regularmente se traduce en insatisfacción y descontento. En el año 2018, de acuerdo con los datos de la wvs-7, la ciudadanía le otorgó una calificación de 5.2, en una escala de 1 a 10 , a la calidad de la democracia mexicana. ${ }^{27}$ Ese mismo año, el proyecto Variedades de la democracia (V-Dem) le asignó una calificación de 0.73 en una escala de 0 a 1 a la democracia electoral en el país (gráfica 1). Como se puede ver, la calificación que la ciudadanía le asigna al nivel de la democracia en el país es, consistentemente, más bajo que el atribuido al país por la metodología del V-Dem. Esta tendencia se ha mantenido por lo menos desde el año 2005, si bien la diferencia entre la opinión pública y el indicador del proyecto V-Dem es más amplia en 2018.

${ }^{27}$ Considerando el diseño complejo de la muestra, el promedio de 5.2 es una estimación puntual con un intervalo de confianza de $95 \%$ entre 5.09 y 5.39 . 


\section{GrÁficA 1}

\section{Satisfacción con la democracia}

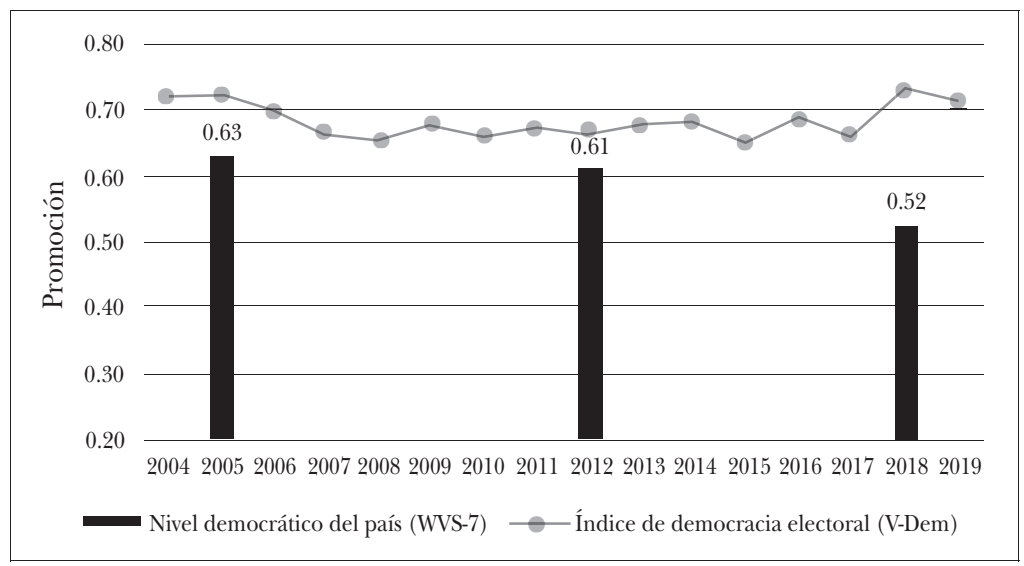

Fuente: elaboración propia con datos de la World Value Survey 7-México y Varieties of Democracy Project (V-Dem).

Además de expresar su descontento con el funcionamiento de la democracia, la ciudadanía mexicana también desconfía de la integridad de los procesos electorales. Hay que decir que, desde hace más de dos décadas, las instituciones de administración electoral han conseguido garantizar certeza en la organización y celebración de las elecciones. ${ }^{28}$ En ese proceso, se han desarrollado significativas capacidades institucionales para responder a los retos logísticos que se incrementan de una elección a otra.

Sin embargo, a pesar de los avances institucionales, persisten importantes desafíos en diversas áreas de la integridad electoral. Por ejemplo, con frecuencia se descalifica la credibilidad de las autoridades electorales, especialmente cuando

${ }^{28}$ Un balance comprensivo se encuentra en los ensayos del siguiente volumen: Instituto Federal Electoral, IFE, Instituto Federal Electoral, 20 años, México, IFE, 2010. En 2014, como resultado de una reforma constitucional, el IFE se transformó en el Instituto Nacional Electoral (INE). 
los comicios han sido controvertidos. ${ }^{29}$ La evidencia muestra, no obstante, que las elecciones presidenciales de 2006, y también las de 2012 en alguna medida, provocaron conflictos que fueron más un producto de la polarización entre los candidatos y sus partidos que de las fallas en la conducción de las elecciones. ${ }^{30}$ Langston ha mostrado, en esa línea, que la constante reforma de leyes e instituciones ha permitido a las elites partidistas imponer límites a la entrada de nuevos competidores y frenar las posibilidades de los órganos reguladores de fiscalizar efectivamente los gastos de campaña. ${ }^{31}$ A la par, los partidos políticos han buscado limitar los alcances regulatorios de las instituciones electorales, lo mismo por medio de una litigación constante que a través de la captura o condicionamiento de sus órganos directivos. ${ }^{32}$

En este contexto, la desconfianza ciudadana en las elecciones se afianzó a lo largo de más de una década. De acuerdo con los datos de la wvs-7, esta tendencia persistía en 2018. Los datos revelan que los ciudadanos calificaron con un 0.49 (el rango de intervalo de confianza de 95\% [IC-95\%]: 0.47, 0.50)

${ }^{29}$ Un plano en el que los desafíos de la integridad electoral han sido especialmente visibles es el de la política subnacional. Véase: Irma Méndez de Hoyos, "Los organos de administración electoral y la calidad de las elecciones locales en México", en Irma Méndez de Hoyos y Nicolás Loza (eds.), Instituciones electorales, opinión pública y poderes políticos locales en México, México, Flacso-México, 2013.

${ }^{30}$ La crisis poselectoral en México, en 2006, también constituye una vívida ilustración de las consecuencias adversas de la desconfianza en las elecciones y la negación de los perdedores a aceptar su derrota. Véanse: Kathleen Bruhn y Kenneth F. Greene, "Elite Polarization Meets Mass Moderation in Mexico's 2006 Elections", PS: Political Science E् amp; Politics 40, núm. 1 (2007); Luis Estrada y Alejandro Poiré, "Learning to lose, taught to protest: Mexico's 2006 election”, Journal of Democracy 18, núm. 1 (2007); Ugues Jr. y Medina Vidal, "Public evaluations...”, art. cit.

${ }^{31}$ Joy Langston, “¿Por qué los partidos hacen trampa? Cambios en las normas electorales en México despúes de la democratización”, Política y Gobierno XxviII, núm. 2 (2020).

${ }^{32}$ Rodrigo Montes de Oca, Mexico's Electoral Authorities: Implications for Democracy and the Rule of Law, Houston, Texas, Rice University's Baker Institute for Public Policy, 2018. 
la integridad de las elecciones mexicanas. La percepción de manipulación de las elecciones, a su vez, alcanzó un puntaje de 0.60 (IC-95\%: 0.59, 0.61).

Poniendo en perspectiva estos datos, la gráfica 2 muestra que México se encuentra en los últimos lugares de percepción ciudadana de integridad electoral en un grupo de países latinoamericanos. ${ }^{33}$ En cambio, destaca cuando se trata de percepción de manipulación electoral. De acuerdo con la opinión pública, las elecciones mexicanas son más propias de un autoritarismo electoral, donde predomina la manipulación sobre la integridad de los comicios. Esto contrasta con los resultados de una encuesta global entre expertos, el Proyecto Integridad Electoral (PIE por su siglas en inglés), donde México obtiene un promedio de 61 sobre 100 a lo largo del periodo 2012-2018. ${ }^{34}$ Por su parte, el proyecto Variedades de la democracia, mediante el "índice de elecciones libres", le da una calificación promedio de 0.77 en una escala de 0 a 1 , también durante el periodo 2012-2018. ${ }^{35}$ En otras palabras, si bien la calidad de las elecciones en el país no llega a alcanzar el nivel más alto de integridad, sí pueden ser consideradas elecciones democráticas -a diferencia de lo que percibe la opinión pública.

Finalmente, la gráfica 3 presenta la distribución de actitudes de interés en la política. Como puede verse, la actitud predominante es el poco interés en asuntos políticos (40\%). No sólo eso, ya que poco menos de una tercera parte de la población se interesa por lo menos algo en la política y menos del 10\% declara que se interesa "mucho".

${ }^{33}$ Los países que se muestran en las gráficas son lo que están incluidos en la base de datos de la séptima ronda de la World Value Survey.

${ }^{34}$ Pippa Norris y Max Grömping, 2019, https://www.electoralintegrityproject.com/

35 Véase: Michael Coppedge et al., V-Dem Codebook v10, Varieties of Democracy (V-Dem) Project, 2020. 


\section{GrÁFICA 2}

Percepción ciudadana de integridad y manipulación electoral en América Latina

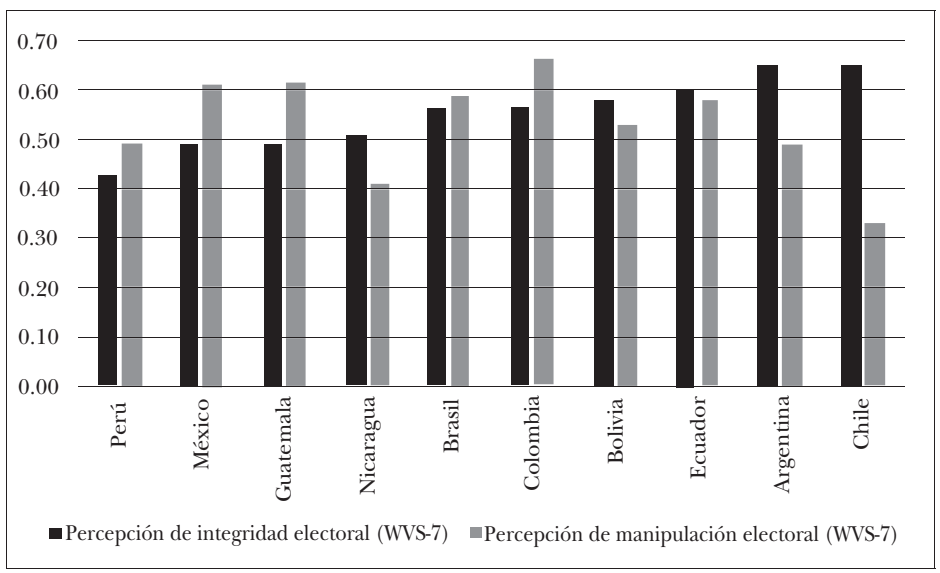

Fuente: elaboración propia con datos de la World Value Survey 7-México. Los países seleccionados son los que forman parte de la muestra de países levantada por el wvs-7 en América Latina.

\section{GRÁFICA 3}

Interés en la política

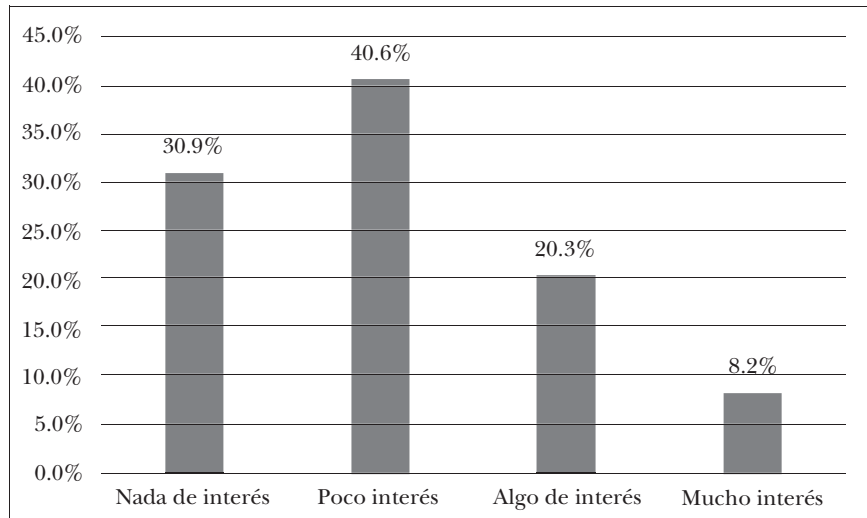

Fuente: elaboración propia con datos de la World Value Survey-7-México, incorporando el diseño muestral. 


\section{Resultados}

La evidencia descriptiva muestra que la ciudadanía mexicana percibe avances limitados en la integridad electoral. Más bien encuentra que diversas prácticas fraudulentas o de manipulación electoral son generalizadas. ¿Influyen las experiencias de los mexicanos con el ciclo electoral en su evaluación del funcionamiento democrático del régimen? Esa evaluación, ¿está condicionada de alguna forma por el interés que tienen en la política? Los resultados del análisis se muestran en el cuadro 1.

\section{Cuadro 1}

Determinantes del nivel de democracia en México

\begin{tabular}{|c|c|c|c|c|}
\hline Variables & M1 & M2 & M3 & M4 \\
\hline \multirow[t]{2}{*}{ Percepción de integridad electoral (PIE) } & $1.67 * * *$ & 1.65 & $1.33^{* * *}$ & 0.72 \\
\hline & {$[0.370]$} & {$[0.870]$} & {$[0.368]$} & {$[0.881]$} \\
\hline \multirow[t]{2}{*}{ Percepción de manipulación electoral (PME) } & $-2.63 * * *$ & -0.44 & $-1.92 * * *$ & 0.23 \\
\hline & {$[0.329]$} & {$[0.793]$} & {$[0.352]$} & {$[0.846]$} \\
\hline \multirow{2}{*}{ Interés en la política (IP) } & 0.11 & $0.77 * *$ & 0.07 & $0.58^{*}$ \\
\hline & {$[0.074]$} & {$[0.239]$} & {$[0.083]$} & {$[0.254]$} \\
\hline \multirow[t]{2}{*}{ PIE $\times$ IP } & & 0.00 & & 0.27 \\
\hline & & {$[0.377]$} & & {$[0.368]$} \\
\hline \multirow[t]{2}{*}{ PME $\times$ IP } & & $-1.07 * *$ & & $-1.04 * *$ \\
\hline & & {$[0.327]$} & & {$[0.347]$} \\
\hline \multirow[t]{2}{*}{ Importancia de las elecciones } & & & -0.13 & -0.09 \\
\hline & & & {$[0.166]$} & {$[0.166]$} \\
\hline \multirow[t]{2}{*}{ Satisfacción económica } & & & $0.10^{* *}$ & $0.10^{* *}$ \\
\hline & & & {$[0.033]$} & {$[0.032]$} \\
\hline \multirow[t]{2}{*}{ Comprensión de la democracia } & & & $-1.59 *$ & $-1.56^{*}$ \\
\hline & & & {$[0.618]$} & {$[0.619]$} \\
\hline \multicolumn{5}{|l|}{ Identificación partidista: PRI } \\
\hline \multirow[t]{2}{*}{ PAN } & & & -0.40 & -0.37 \\
\hline & & & {$[0.320]$} & {$[0.320]$} \\
\hline \multirow[t]{2}{*}{ PRD } & & & -0.27 & -0.31 \\
\hline & & & {$[0.582]$} & {$[0.570]$} \\
\hline \multirow[t]{2}{*}{ Verde } & & & $-1.74 *$ & $-1.67 *$ \\
\hline & & & {$[0.746]$} & {$[0.704]$} \\
\hline
\end{tabular}




\section{CuAdro 1 (conclusión)}

\section{Determinantes del nivel de democracia en México}

\begin{tabular}{|l|c|c|c|c|}
\hline Morena & & & $-0.73^{*}$ & $-0.68^{*}$ \\
\hline & & & {$[0.288]$} & {$[0.287]$} \\
\hline No-partidista & & & $-0.53^{*}$ & $-0.53^{*}$ \\
\hline & & & {$[0.265]$} & {$[0.264]$} \\
\hline Antipartidista & & & $-0.72^{*}$ & $-0.70^{*}$ \\
\hline & & & {$[0.285]$} & {$[0.285]$} \\
\hline No sabe & & & $-0.76^{* *}$ & $-0.74 * *$ \\
\hline & & & {$[0.284]$} & {$[0.284]$} \\
\hline Ideología & & & $0.12^{* * *}$ & $0.12 * * *$ \\
\hline & & & {$[0.031]$} & {$[0.031]$} \\
\hline Consumo informativo & & & $-0.18^{* *}$ & $-0.17 * *$ \\
\hline & & & {$[0.057]$} & {$[0.056]$} \\
\hline Organizaciones sociales & & & $0.43^{*}$ & $0.46^{* *}$ \\
\hline & & & {$[0.172]$} & {$[0.172]$} \\
\hline Mujer & & & 0.23 & 0.24 \\
\hline & & & {$[0.144]$} & {$[0.145]$} \\
\hline Edad & & & $-0.02 * *$ & $-0.02 * *$ \\
\hline & & & {$[0.005]$} & {$[0.005]$} \\
\hline Escolaridad & & & $-0.12^{*}$ & $-0.12^{*}$ \\
\hline & & & {$[0.055]$} & {$[0.055]$} \\
\hline Rango de ingreso & & & $0.19^{* * *}$ & $0.18^{* * *}$ \\
\hline & & & {$[0.033]$} & {$[0.033]$} \\
\hline Localidad urbana & & & $-0.35^{*}$ & $-0.35^{*}$ \\
\hline & & & {$[0.157]$} & {$[0.156]$} \\
\hline Constante & $5.83^{* * *}$ & $4.49^{* * *}$ & $6.59^{* * * *}$ & $5.46^{* * *}$ \\
\hline & {$[0.283]$} & {$[0.556]$} & {$[0.689]$} & {$[0.877]$} \\
\hline N= & 1,577 & 1,577 & 1,437 & 1,437 \\
\hline R cuadrada & 0.047 & 0.053 & 0.133 & 0.138 \\
\hline & & & & \\
\hline
\end{tabular}

Modelos de regresión lineal ajustando por el diseño muestral complejo. Errores estándar linearizados entre paréntesis. Niveles de significancia: *p $<.05, * * \mathrm{p}<.01, * * * \mathrm{p} .001$

El modelo 1 presenta los resultados de una regresión que evalúa los efectos principales de las variables independientes 
en la dependiente, excluyendo las variables de control. El modelo 2 introduce el término de interacción, sin incluir todavía las variables de control. Los modelos 3 y 4 replican el análisis de los modelos previos, pero contemplan todas las variables relevantes. En específico, el modelo 3 muestra que la evidencia confirma las hipótesis 1 y 2, relativas al efecto directo de las percepciones de integridad y manipulación electoral en la satisfacción con la democracia. De acuerdo con los resultados de este modelo, la percepción de integridad ejerce una influencia positiva en la variable dependiente, a diferencia de la percepción de manipulación, que tiene el efecto opuesto.

Para ilustrar estos hallazgos, la gráfica 4 presenta los valores predichos del nivel de satisfacción con la democracia en el país cuando la percepción de integridad pasa de su nivel mínimo a su nivel máximo y el resto de las variables se mantienen en su valor medio. Según este análisis, si la percepción media de la integridad electoral fuera igual a cero -es decir, ningún votante encontrara algún indicio de integridad-, los mexicanos calificarían la calidad democrática del sistema con un 4.6 (el rango del intervalo de confianza de 95\% [IC95\%] es: 4.3, 5). En la situación opuesta, si el valor medio fuera igual a 1, representando el máximo nivel posible de integridad electoral, entonces la calidad de la democracia mexicana sería calificada con 5.97 (IC95\%: $5.58,6.37)$. De igual manera, la gráfica 4 muestra qué tanto cambia la satisfacción con la democracia cuando la percepción de manipulación electoral pasa de su nivel mínimo al máximo. En este caso, como se trata de una relación negativa, los ciudadanos le darían una calificación de 6.5 (IC95\%: $6,6.9)$, si no hubiera ningún indicio de fraude o malas prácticas en las elecciones. Si la opinión general es que la manipulación es predominante, la calificación desciende hasta 4.5 (IC95\%: 4.2, 4.9). 


\section{GrÁFICA 4}

Satisfacción con la democracia: predicciones marginales

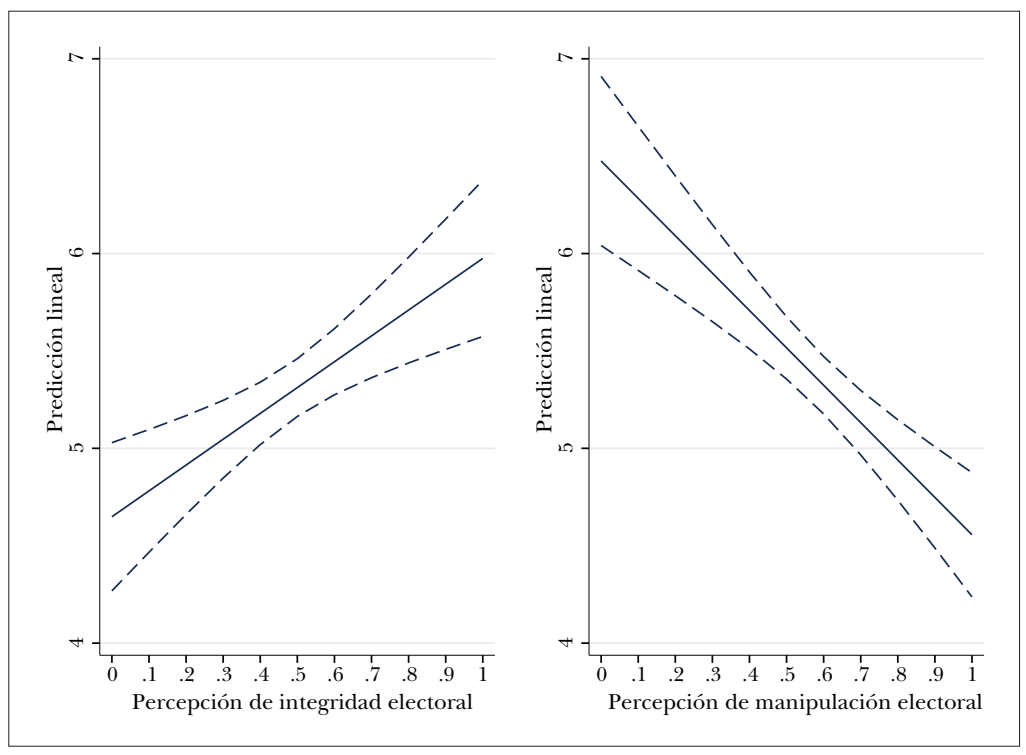

Fuente: predicciones basadas en el modelo м3, manteniendo el resto de las variables en su valor medio. Intervalos de confianza de $95 \%$.

Por otra parte, el modelo 3 revela que la relación directa entre el interés en la política y la satisfacción con el funcionamiento de este régimen no es estadísticamente significativa. Lo es si se omiten las variables de control, como en el modelo 1. Una vez que se controlan los efectos de otros predictores, esa relación deja de ser sistemática. Según el análisis, por mucho interés que se tenga en los asuntos públicos, el desempeño democrático del sistema político mexicano no necesariamente será satisfactorio.

El modelo 4 presenta los resultados de la evaluación de la tercera y cuarta hipótesis. Los datos muestran que la tercera hipótesis carece de respaldo empírico. Esta hipótesis es la que se refiere a la relación condicional entre el interés en la política y la percepción de integridad. Aunque el coeficiente del 
término de interacción tiene un signo positivo, como se esperaba, no alcanza a ser estadísticamente significativo de acuerdo con el estándar usual ( $p>0.05)$. En otros términos, los datos indican que el efecto de la percepción de integridad electoral en la satisfacción con la democracia es independiente de qué tanto los ciudadanos se interesen o no en la política.

En contraste, los resultados del modelo 4 sí ofrecen respaldo a la cuarta hipótesis. De acuerdo con el coeficiente del término de interacción, la relación entre la percepción de manipulación electoral y la satisfacción con la democracia se ve modificada por el grado de interés que la ciudadanía tiene en la política. El efecto condicional es negativo, lo cual implica que si la percepción de manipulación electoral es elevada, la satisfacción con la democracia será menor, especialmente entre quienes estén muy interesados en la política.

Para ilustrar esta relación, la gráfica 5 muestra el nivel de satisfacción con la democracia, esperado a lo largo de diversos valores en la escala de percepción de manipulación electoral, bajo dos condiciones. En una, se asume que el interés en la política es nulo y en otra, que es muy alto. Los valores en la escala de percepción de manipulación electoral corresponden a valores sustantivos observados en la población mexicana. En la muestra correspondiente, con los datos ya ponderados, el $70 \%$ de los mexicanos tiene percepción de manipulación electoral entre un puntaje mínimo de 0.34 y un máximo 0.8 en una escala de 0 a 1 . Entonces, si el interés en la política es "mucho", la satisfacción con la democracia esperada cuando la percepción de manipulación electoral es baja (0.34) es de 6.5 (IC95\%: 6.0, 7.0). Ese nivel de satisfacción disminuye a 4.7 (IC95\%: 4.3, 5.1) si la percepción de manipulación electoral alcanza un nivel mayor (0.8). Esta diferencia es estadísticamente significativa -como se puede apreciar en la pendiente y en el hecho de que los intervalos de confianza no se traslapan-. En el caso opuesto, cuando no existe ningún interés en la política, no se observa un cambio sustantivo en la satisfacción con la democracia, independientemente de qué tanto se perciba manipulación electoral. Si 
esta percepción se encuentra en un bajo nivel, la satisfacción con la democracia tendrá una calificación de 5.4 (IC95\%: $5.1,5.8)$ y permanecerá estable, aunque aumente la percepción de manipulación electoral (5.0, Ic95\%: 4.7, 5.4).

\section{Gráfica 5}

Influencia condicional del interés en la política en la relación de la percepción de manipulación electoral y la satisfacción democrática

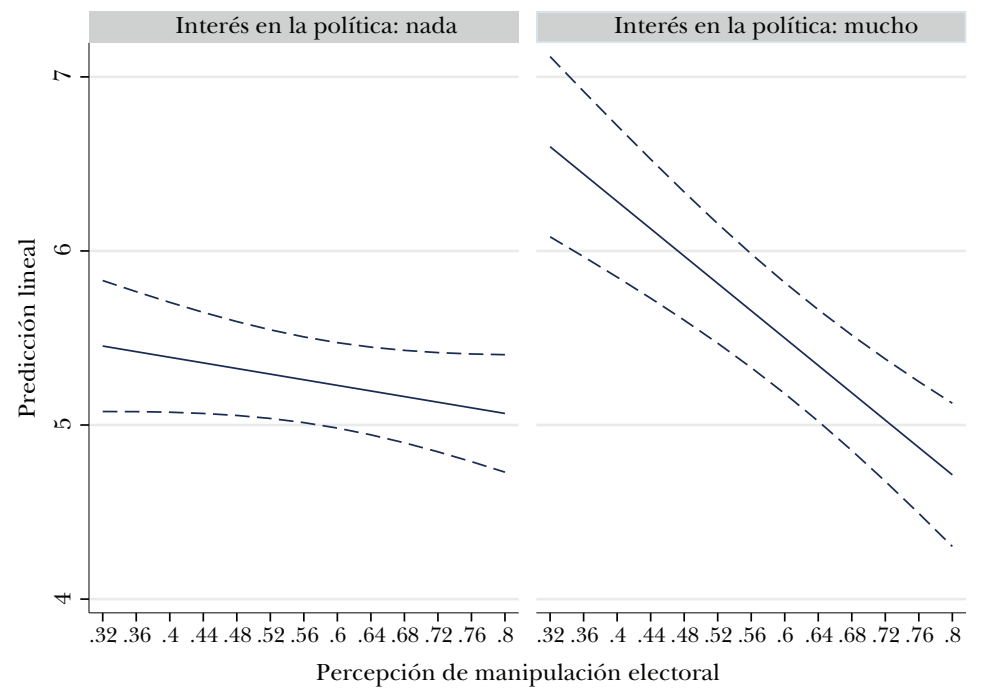

Fuente: predicciones basadas en el modelo $\mathrm{m} 4$, manteniendo al resto de las variables en su valor medio. Intervalos de confianza de $95 \%$.

Es preciso tener presente que las interacciones estadísticas producen efectos simétricos, de manera que las percepciones de manipulación electoral también condicionan la relación entre el interés en la política y la satisfacción con la democracia (véanse los dos paneles de la gráfica Al en el apéndice). En este caso, se encuentra que la satisfacción con la democracia entre las personas que tienen un alto interés en 
la política es mayor, a condición de que perciban una baja o nula manipulación de las elecciones. Así, conforme aumenta su percepción de que las malas prácticas se extienden, su satisfacción con la democracia disminuye significativamente.

$\mathrm{Al}$ especificar el modelo, se tuvo en cuenta la importancia de disminuir el riesgo de omitir alguna variable que pudiera confundir el efecto de las percepciones de integridad o manipulación electoral. ${ }^{36}$ Con ese propósito, la prueba de hipótesis contempla 12 variables de control, además de las tres variables independientes y el término de interacción. Como se observa en el modelo 4 , los resultados son robustos a la inclusión de todas las variables de control. A la par, se verificó que las estimaciones no se vieran afectadas por problemas de multicolinealidad. Aun en los modelos que incluyen los efectos directos de todas las variables independientes y de control, se encontró que el factor de inflación de la varianza promedio no llega a ser mayor a 1.44.

Con todo, las inferencias con datos de encuestas de opinión pública suelen enfrentarse al problema de la causalidad inversa. Teniendo presente este riesgo, las hipótesis de este estudio se formularon a partir de los antecedentes en la teoría y los hallazgos empíricos acerca de la evaluación de la democracia. En este campo, la evidencia respalda el carácter inductivo de los juicios ciudadanos acerca del sistema político. Es decir, la opinión pública acerca de las instituciones o de la democracia se origina en la acumulación de experiencias concretas e información específica. ${ }^{37}$ Por esta razón, es

${ }^{36} \mathrm{Al}$ evaluar las hipótesis, es usual analizar los coeficientes de las variables y el significado sustantivo de sus efectos, independientemente del porcentaje de variación explicada por el coeficiente de determinación ( $\mathrm{R}$ cuadrada). Con todo, como recomienda un revisor, la $\mathrm{R}$ cuadrada contribuye a poner en perspectiva la relevancia teórica de los modelos. En este caso, las hipótesis analizadas dan cuenta del 5.3\% de la variación de la satisfacción con la democracia (cuadro 1, modelo 2). Dado que el modelo en su conjunto equivale al $13.8 \%$ (modelo 4), las hipótesis de este estudio representan el $38 \%$ de esa proporción.

37 David Easton, "A Re-assessment of the Concept of Political Support", British Journal of Political Science 5, núm. 4 (1975); Pippa Norris, "The 
difícil que la satisfacción con la democracia sea la causa de que las personas crean que los votos se cuentan más o menos limpiamente o que persisten prácticas de compra y coacción del voto. Es más coherente pensar que la gente decide si el sistema es más o menos democrático a partir de la información que tiene, por experiencias personales o indirectas, acerca de los atributos que componen los índices de integridad y manipulación de las elecciones.

\section{DisCUSIÓN Y CONCLUSIONES}

Este estudio analizó el papel que desempeña el interés en la política en la relación entre las percepciones de integridad y manipulación en la satisfacción con la democracia. El argumento formulado establecía que los ciudadanos que más se interesan en la política son quienes se verían más influidos por sus experiencias con la calidad del proceso electoral. Se esperaba que la evidencia de honestidad e imparcialidad en las elecciones aumentara la satisfacción con la democracia de las personas más interesadas en la política. Asimismo, se esperaba que si esas personas detectaban manipulación o prácticas electorales fraudulentas, su evaluación del funcionamiento del sistema sería particularmente negativo. En contraste, los ciudadanos poco interesados en los asuntos públicos se verían menos afectados por la integridad de los procesos electorales.

Los resultados del análisis confirman parcialmente las expectativas iniciales. Por un lado, confirman que la percepción de integridad electoral influye positivamente en la satisfacción con la democracia, mientras que la percepción de manipulación de las elecciones provoca la reacción opuesta. En este sentido, la opinión de la ciudadanía acerca de la in-

Conceptual Framework of Political Support", en Tom W.G. van der Meer y Sonja Zmerli (eds.), Handbook on Political Trust, Reino Unido, Edward Elgar Publishing, 2017. 
tegridad o manipulación del proceso electoral resulta ser un elemento clave de la evaluación del desempeño del sistema democrático. Por otra parte, el análisis indica que la relación entre la percepción de integridad electoral y la satisfacción con la democracia no está condicionada por el interés en la política. Si bien este resultado obliga a descartar una de las hipótesis principales, también permite destacar la centralidad de la percepción de integridad electoral en la evaluación del desempeño del régimen. A saber, sin importar qué tanto se interese una persona en la política, en la medida en que perciba que las elecciones son libres y limpias, su opinión sobre el desempeño democrático del sistema será favorable.

En cambio, los resultados respaldan la hipótesis de que el interés en la política condiciona negativamente el efecto de la percepción de manipulación electoral en la satisfacción con el régimen. La percepción de prácticas fraudulentas durante las elecciones, en sí misma, disminuye la satisfacción con la democracia. Sin embargo, las personas que más se interesan en la política son quienes reaccionan con mayor insatisfacción ante la manipulación electoral. En ese sentido, el interés en la política sí marca una diferencia. Quienes más se interesan en la política le dan más importancia a que el proceso no se vea distorsionado por intentos deliberados de favorecer a ciertos contendientes a costa de otros. Cuando detectan que el proceso es menos limpio o imparcial de lo que debería ser, juzgan más severamente el funcionamiento de la democracia.

La principal contribución de este estudio es mostrar que el efecto negativo de las percepciones de manipulación electoral en la satisfacción con la democracia es más pronunciado entre los ciudadanos que más se interesan en la política. Son las personas más interesadas en los asuntos públicos quienes más reaccionan negativamente ante la evidencia de manipulación electoral -comparadas con quienes tienen menos interés en la política-. Se puede decir que, en materia de integridad electoral, el interés en la política favorece que las personas sean más exigentes con la limpieza del pro- 
ceso, pues son quienes más esperan que se minimicen la manipulación y las malas prácticas.

Es preciso señalar que estos hallazgos son aplicables, en principio, al caso de México, que es interesante por la brecha persistente entre la calidad de las elecciones y la percepción de integridad electoral entre los ciudadanos. Este artículo muestra que la mayoría de los votantes mexicanos expresa escepticismo acerca de la integridad electoral y tiende a creer que prevalecen las prácticas de manipulación de las elecciones. Incluso en este contexto de desconfianza, la percepción de integridad influye positivamente en la satisfacción con la democracia. Al mismo tiempo, la evidencia sugiere que un segmento del electorado mexicano, caracterizado por un mayor interés en la política, tiene expectativas más exigentes acerca de la calidad de las elecciones. Esto implica que, en la medida en que los procesos electorales se vean inmersos en controversias o afectados por la violencia del crimen organizado, no sólo la ciudadanía en su conjunto se verá menos afecta a la democracia, sino que los ciudadanos más proclives a involucrase en la política serán los más insatisfechos con el desempeño del sistema.

Los resultados de este estudio, al propio tiempo, podrían generalizarse más allá del caso mexicano. En futuros estudios es factible esperar, en principio, resultados similares en contextos similares. Es decir, democracias electorales en desarrollo, cuyas elecciones cumplen con mínimos de integridad, pero en las que persisten malas prácticas derivadas de capacidades institucionales insuficientes y el comportamiento oportunista de los agentes políticos. Sin embargo, el efecto condicional del interés en la política puede ser igualmente relevante en democracias desarrolladas, en las que se supone que el fraude y las malas prácticas son virtualmente inexistentes. En esos casos, la ciudadanía más involucrada en la política también podría reaccionar negativamente ante la evidencia de manipulación electoral. Asimismo, el interés político podría condicionar positivamente la percepción de integridad electoral en democracias débiles o autoritarismos electorales. 
Precisamente porque se trata de indicios de democratización, la satisfacción de los ciudadanos más interesados en la política podría responder favorablemente a la evidencia de integridad electoral.

\section{ApÉNDICE}

\section{Cuadro A1 \\ Variables: estadística descriptiva}

\begin{tabular}{lccccc}
\hline Variable dependiente & Obs & Media & Desviación típica & Mínimo & Máximo \\
\hline $\begin{array}{l}\text { Satisfacción con la } \\
\quad \text { democracia }\end{array}$ & 1728 & 5.23 & 2.8 & 1 & 10 \\
& & & & & \\
$\quad \begin{array}{l}\text { Variables independientes } \\
\text { Percepción de integridad }\end{array}$ & & & & & \\
$\quad$ electoral & 1726 & 0.49 & 0.2 & 0 & 1 \\
$\begin{array}{l}\text { Percepción de manipulación } \\
\text { electoral }\end{array}$ & 1725 & 0.61 & 0.2 & 0 & 1 \\
Interés en la política & 1738 & 2.06 & 0.9 & 1 & 4 \\
& & & & & \\
Variables de control & & & & & \\
Importancia de las elecciones & 1735 & 0.64 & 0.48 & 1 & 10 \\
Satisfacción económica & 1738 & 6.81 & 2.4 & 1 & 10 \\
Comprensión de la democracia & 1622 & 0.56 & 0.13 & 1 & 10 \\
Identificación partidista: PRI & 1651 & 0.11 & 0.3 & 0 & 1 \\
$\quad$ PAN & & 0.09 & 0.3 & 0 & 1 \\
$\quad$ PRD & & 0.02 & 0.1 & 0 & 1 \\
$\quad$ Verde & & 0.00 & 0.1 & 0 & 1 \\
$\quad$ Morena & & 0.16 & 0.4 & 0 & 1 \\
$\quad$ No partidista & & 0.26 & 0.4 & 0 & 1 \\
$\quad$ Antipartidista & & 0.17 & 0.4 & 0 & 1 \\
$\quad$ No sabe & & 0.19 & 0.4 & 0 & 1 \\
Ideología & 1738 & 5.14 & 3.1 & 1 & 4 \\
Consumo informativo & 1738 & 2.69 & 1.5 & 1 & 4 \\
Membresía en organizaciones & & & & & \\
$\quad$ sociales & 1738 & 0.31 & 0.43 & 1 & 4 \\
\hline
\end{tabular}




\section{Cuadro A1 (conclusión) \\ Variables: estadística descriptiva}

\begin{tabular}{lrrrrr}
\hline Sexo: mujer & 1739 & 0.50 & 0.5 & 0 & 1 \\
Edad & 1737 & 43.33 & 16.7 & 18 & 90 \\
Escolaridad & 1731 & 3.03 & 1.7 & 0 & 8 \\
Rango de ingreso & 1717 & 4.23 & 2.4 & 1 & 10 \\
Localidad: urbana & 1739 & 0.69 & 0.5 & 0 & 1 \\
\hline
\end{tabular}

Cuadro A2

Percepciones de integridad y manipulación electoral: proporciones

\begin{tabular}{|c|c|c|c|c|c|}
\hline & $\begin{array}{l}\text { Nada } \\
\text { frecuente }\end{array}$ & $\begin{array}{l}\text { Poco } \\
\text { frecuente }\end{array}$ & $\begin{array}{l}\text { Algo } \\
\text { frecuente }\end{array}$ & $\begin{array}{l}\text { Muy } \\
\text { frecuente }\end{array}$ & Total \\
\hline \multicolumn{6}{|l|}{ Percepción de integridad electoral } \\
\hline \multicolumn{6}{|l|}{ Los votos se cuentan } \\
\hline limpiamente & 0.34 & 0.33 & 0.20 & 0.12 & 1.00 \\
\hline $\begin{array}{l}\text { Los periodistas hacen una } \\
\text { cobertura objetiva de las }\end{array}$ & & & & & \\
\hline elecciones & 0.17 & 0.25 & 0.24 & 0.33 & 1.00 \\
\hline Las autoridades electorales & & & & & \\
\hline son justas & 0.34 & 0.34 & 0.19 & 0.13 & 1.00 \\
\hline $\begin{array}{l}\text { Los votantes tienen opciones } \\
\text { reales entre las cuales elegir }\end{array}$ & 0.19 & 0.25 & 0.25 & 0.31 & 1.00 \\
\hline $\begin{array}{l}\text { Las mujeres tienen igualdad } \\
\text { de oportunidades para ser } \\
\text { candidatas a puestos de } \\
\text { elección popular }\end{array}$ & 0.17 & 0.25 & 0.24 & 0.34 & 1.00 \\
\hline $\begin{array}{l}\text { Percepción de manipulación } \\
\text { electoral }\end{array}$ & & & & & \\
\hline $\begin{array}{l}\text { Se prohíbe competir a } \\
\text { candidatos de la oposición }\end{array}$ & 0.27 & 0.34 & 0.24 & 0.15 & 1.00 \\
\hline $\begin{array}{l}\text { Los noticieros de televisión } \\
\text { favorecen al partido }\end{array}$ & & & & & \\
\hline gobernante & 0.14 & 0.20 & 0.28 & 0.38 & 1.00 \\
\hline Hay compra de votos & 0.09 & 0.13 & 0.17 & 0.62 & 1.00 \\
\hline Los ricos compran las elecciones & 0.13 & 0.17 & 0.23 & 0.47 & 1.00 \\
\hline
\end{tabular}


CuAdro A2 (conclusión)

Percepciones de integridad y manipulación electoral: proporciones

\begin{tabular}{llllll}
\hline $\begin{array}{l}\text { Se amenaza a los votantes con } \\
\text { violencia en las urnas }\end{array}$ & 0.22 & 0.23 & 0.26 & 0.29 & 1.00 \\
\hline
\end{tabular}

Fuente: elaboración propia con datos de la World Value Survey 7-México incorporando el diseño muestral.

\section{GRÁFICA A1}

Satisfacción con la democracia: la interacción entre la percepción de manipulación electoral y el interés en la política

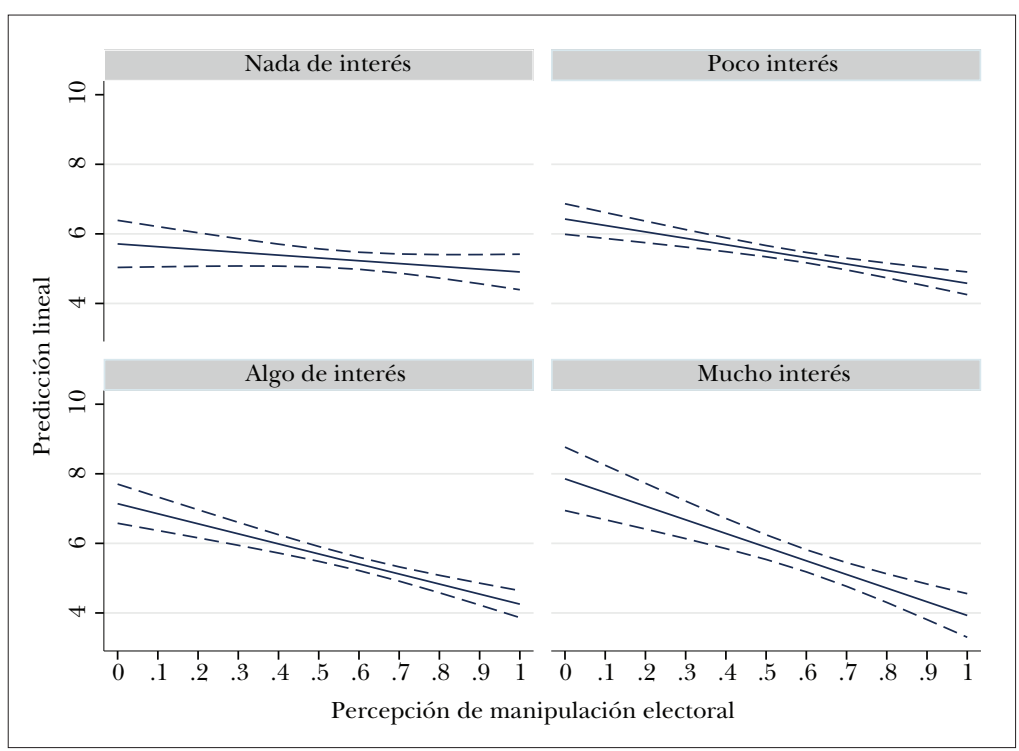




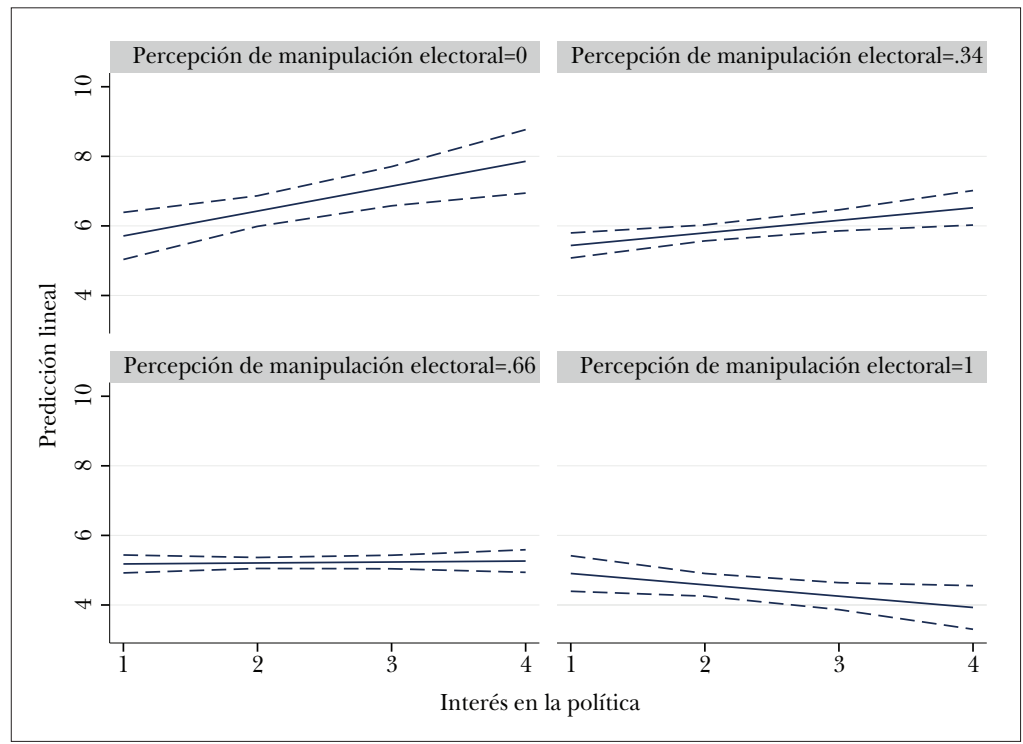

Fuente: predicciones basadas en el modelo $\mathrm{M} 4$, manteniendo el resto de las variables en su valor medio. Intervalos de confianza de $95 \%$.

\section{REFERENCIAS BIBLIOGRÁFICAS}

Anderson, Christopher J., André Blais, Shaun Bowler, Todd Donovan y Ola Listhaug, Loser's Consent: Elections and Democratic Legitimacy, Oxford, University Press, 2005.

Birch, Sarah, "Electoral Institutions and Popular Confidence in Electoral Processes: A Cross-National Analysis", Electoral Studies 27, núm. 2 (2008, pp.: 305-320, https:/ /doi.org/10.1016/j. electstud.2008.01.005

Bishop, Sylvia y Anke Hoeffler, "Free and Fair Elections: A New Database", Journal of Peace Research 53, núm. 4 (2016), pp. 608616, https://doi.org/10.1177/0022343316642508

Bоoтh, John A. y Mitchell A. Seligson, The Legitimacy Puzzle in Latin America: Political Support and Democracy in Eight Nations, Cambridge, Nueva York, Cambridge University Press, 2009. 
Bowler, Shaun, Thomas Brunell, Todd Donovan y Paul Gronke, "Election Administration and Perceptions of Fair Elections", Electoral Studies 38 (2015), pp. 1-9, https://doi.org/10.1016/j. electstud.2015.01.004

Bruhn, Kathleen y Kenneth F. Greene, "Elite Polarization Meets Mass Moderation in Mexico's 2006 Elections", PS: Political Science Ẽamp; Politics 40, núm. 1 (2007), pp. 33-38, https://doi. org/10.1017/S1049096507070060

CHo, Youngho, "To Know Democracy Is to Love It: A Cross-National Analysis of Democratic Understanding and Political Support for Democracy", Political Research Quarterly 67, núm. 3 (2014), pp. 478-488, https://doi.org/10.1177/1065912914532721

Coppedge, Michael, John Gerring, Carl Henrik Knutsen, Staffan I. Lindberg, Jan Teorell, David Altman, Michael Bernhard et al., V-Dem Codebook V10. Varieties of Democracy (V-Dem) Project., 2020.

Diamond, Larry, Developing Democracy: Toward Consolitadion, Baltimore, The John Hopkins University Press, 1999.

Easton, David, "A Re-assessment of the Concept of Political Support”, British Journal of Political Science 5, núm. 4 (1975), pp. 435-457, https://doi.org/10.1017/S0007123400008309 ElkLit, J. y A. Reynolds, "The Impact of Election Administration on the Legitimacy of Emerging Democracies: A New Comparative Politics Research Agenda", Commonwealth E Comparative Politics 40, núm. 2 (2002), pp. 86-119, https://doi.org/10.1080/ 713999584

Estrada, Luis y Alejandro Poiré, "Learning to Lose, Taught to Protest: Mexico's 2006 Election”, Journal of Democracy 18, núm. 1 (2007), pp. 73-87, https://www.journalofdemocracy.org/ar ticles/the-mexican-standoff-taught-to-protest-learning-to-lose/

Fortin-Rittberger, Jessica, Philipp Harfst y Sarah C. Dingler, "The Costs of Electoral Fraud: Establishing the Link between Electoral Integrity, Winning an Election, and Satisfaction with Democracy", Journal of Elections, Public Opinion and Parties 27, núm. 3 (2017): 350-368, https://doi.org/10.1080/17457289. 2017.1310111 
Garnett, Holly Ann, "On the Front Lines of Democracy: Perceptions of Electoral Officials and Democratic Elections", Democratization 26, núm. 8 (2019), pp. 1399-1418, https://doi.org/1 $0.1080 / 13510347.2019 .1641797$

Haerpfer, Christian, Ronald Inglehart, Alejandro Moreno, Christian Welzel, Kseniya Kizilova, Jaime Diez-Medrano, Marta LAGos et al., "World Values Survey: Round Seven-Country-Pooled Datafile”, Madrid y Viena, JD Systems Institute \& wvSA Secretariat, 2020.

Ham, Carolien van, "Getting Elections Right? Measuring Electoral Integrity”, Democratization 22, núm. 4 (2015), pp. 714-737, https://doi.org/10.1080/13510347.2013.877447

Instituto Federal Electoral, IFE, Instituto Federal Electoral, 20 Años, México, IFE, 2010.

Jacobs, Lawrence R., Fay Lomax Cook y Michael X. Delli CarPINI, Talking Together: Public Deliberation and Political Participation in America, Chicago, University of Chicago Press, 2009.

Kerr, Nicholas y Anna LÜhrmann, "Public Trust in Manipulated Elections: The Role of Election Administration and Media Freedom”, Electoral Studies 50 (2017), pp. 50-67, https://doi. org/10.1016/j.electstud.2017.08.003

LAnGSTON, Joy, “¿Por qué los partidos hacen trampa? Cambios en las normas electorales en México despúes de la democratización”, Política y Gobierno XXVIII, núm. 2 (2020), pp. 1-32, http:/ /www.politicaygobierno.cide.edu/index.php/pyg/ar ticle/view/1289

Linde, Jonas, "Why Feed the Hand That Bites You? Perceptions of Procedural Fairness and System Support in Post-Communist Democracies", European Journal of Political Research 51, núm. 3 (2012), pp. 410-434, https://doi.org/10.1111/j.1475-6765.20 11.02005.x

Linde, Jonas y Joakim Ekman, "Satisfaction with Democracy: A Note on a Frequently Used Indicator in Comparative Politics", European Journal of Political Research 42, núm. 3 (2003), pp. 391408, https://doi.org/10.1111/1475-6765.00089 
Luskin, Robert C., "Explaining Political Sophistication", Political Behavior 12, núm. 4 (1990), pp. 331-361, https://doi.org/10.1007/ BF00992793

Magalhães, Pedro C., "Economic Evaluations, Procedural Fairness, and Satisfaction with Democracy", Political Research Quarterly 69, núm. 3 (2016), pp. 522-534, https:/ / doi.org/10.1177\% 2F1065912916652238

Mauk, Marlene, "Electoral Integrity Matters: How Electoral Process Conditions the Relationship between Political Losing and Political Trust", Quality E Quantity, 2020, https:/ / doi.org/10.1007/ s11135-020-01050-1

McAllister, Ian y Stephen White, "Electoral Integrity and Support for Democracy in Belarus, Russia, and Ukraine", Journal of Elections, Public Opinion and Parties 25, núm. 1 (2015), pp. 78-96, https://doi.org/10.1080/17457289.2014.911744

Meer, Tom W.G. van der y Sonja Zmerli, "The Deeple Rooted Concern with Political Trust" (pp. 1-18), en Tom W.G. van der Meer y Sonja Zmerli (eds.), Handbook on Political Trust, Reino Unido, Edward Elgar Publishing, 2017.

MÉndez de Hoyos, Irma, "Los órganos de administración electoral y la calidad de las elecciones locales en México” (pp. 27-58), en Irma Méndez de Hoyos y Nicolás Loza (eds.), Instituciones electorales, opinión pública y poderes políticos locales en México, México, Flacso-México, 2013.

Méndez de Hoyos, Irma, Ferrán Martínez i Coma y Nicolás Loza, "Introducción" (pp. 7-16), en Irma Méndez, Nicolás Loza y Ferrán Martínez (eds.), Integridad electoral: México en perspectiva global, México, Flacso-México, 2019.

Mendizábal, Yuritzi y Alejandro Moreno, "La confianza electoral: el IFE y los partidos políticos” (pp. 227-247), en Alejandro Moreno (ed.), La confianza en las instituciones, México, Centro de Estudios Sociales y de Opinión Pública-Cámara de Diputados, 2010.

Monsiváis Carrillo, Alejandro, "La insatisfacción con la democracia en México (2014-2017)", Perfiles Latinoamericanos 27, núm. 53 (2019), https://doi.org/10.18504/pl2753-012-2019 
Montes De OcA, Rodrigo, Mexico's Electoral Authorities: Implications for Democracy and the Rule of Law, Houston, Rice University's Baker Institute for Public Policy, 2018.

Moreno, Alejandro, "Citizen's Values and Beliefs toward Politics: Is Democracy Growing Attitudinal Roots?” (pp. 29-49), en Andrew Seele y Jacqueline Peschard (eds.), Mexico's Democratic Challenges: Politics, Government, and Society, Washington/Standford, Woodrow Wilson Center Press, Sanford University Press, 2010.

Moreno, Alejandro y Shannon K. O’Neil, "El malestar democrático en México" (pp. 317-324), en Gustavo Meixueiro y Alejandro Moreno (eds.), El comportamiento electoral mexicano en las elecciones de 2012, México, CEsop-Cámara de Diputados (LXII Legislatura), 2014.

Norris, Pippa, "The Conceptual Framework of Political Support" (pp. 19-32), en Tom W.G. van der Meer y Sonja Zmerli (eds.), Handbook on Political Trust, Reino Unido, Edward Elgar Publishing, 2017.

Norris, Pippa, Democratic Deficit: Critical Citizens Revisited, Cambridge, University Press, 2011.

Norris, Pippa, "Do Perceptions of Electoral Malpractice Undermine Democratic Satisfaction? The US in Comparative Perspective”, International Political Science Review 40, núm. 1 (2019), pp. 5-22, https://doi.org/10.1177/0192512118806783

Norris, Pippa, Why Electoral Integrity Matters, Cambridge, University Press, 2014, https://doi.org/10.1017/CBO9781107280861

NorRis, Pippa y Max GrömPing, "Electoral Integrity Worldwide", The Electoral Integrity Project, Australia, University of Sidney, 2019.

Ortega, Reynaldo Yunuen y María Fernanda Somuano, Confianza y cambio político en México: contiendas electorales y el IFE, México, El Colegio de México, 2015.

Pastor, Robert A., "The Role of Electoral Administration in Democratic Transitions: Implications for Policy and Research", Democratization 6, núm. 4 (1999), pp. 1-27, https://doi.org/10.1080/ 13510349908403630 
Pérez-Verduzco, Germán, "Confianza en el Instituto Nacional Electoral mexicano: una perspectiva comparada", Reflexión Política 22, núm. 45 (2020), pp. 103-115, https://doi.org/10.2 9375/01240781.3903

Prior, Markus, "You've Either Got it or You Don't? The Stability of Political Interest over the Life Cycle", The Journal of Politics 72, núm. 3 (2010), pp. 747-766, https://doi.org/10.1017/s002238 1610000149

Robison, Joshua, “The Social Rewards of Engagement: Appealing to Social Motivations to Stimulate Political Interest at High and Low Levels of External Efficacy", Political Studies 65, núm. 1 (2017),pp. 24-41, https://doi.org/10.1177/0032321715619431

Schedler, Andreas, The Politics of Uncertainty: Sustaining and Subverting Electoral Authoritarianism, Oxford, University Press, 2013. Somuano, María Fernanda, "Los determinantes de la legitimidad gubernamental: el sexenio de Enrique Peña Nieto", Foro Internacional60, núm. 2 (2020), pp. 367-396, https://doi.org/10.24 201/fi.v60i2.2729

SONNLeitner, Willibald, La fábrica de la (des)confianza ciudadana: las percepciones cambiantes de la integridad electoral en México, México: Cesop-Cámara de Diputados, 2016.

Ugues Jr., Antonio, “Citizens' Views on Electoral Governance in Mexico", Journal of Elections, Public Opinion and Parties 20, núm. 4 (2010), pp. 495-527, https://doi.org/10.1080/17457 289.2010.512842

Ugues Jr., Antonio, "Public Perceptions of Clean Elections in Mexico: An Analysis of the 2000, 2006, and 2012 Elections", Journal of Politics in Latin America 10, núm. 2 (2018), pp. 77-98, https://doi.org/10.1177/1866802x1801000203

Ugues Jr., Antonio y D. Xavier Medina Vidal, "Public Evaluations of Electoral Institutions in Mexico: An Analysis of the Ife and Trife in the 2006 and 2012 Elections", Electoral Studies 40 (2015), pp. 231-244, https://doi.org/10.1016/j.electstud.2015.09.005

Verba, Sidney, Kay Lehman Schlozman y Henry E. Brady, Voice and Equality: Civic Voluntarism in American Politics, Harvard, University Press, 1995. 
Wegscheider, Carsten y Toralf Stark, "What Drives Citizens' Evaluation of Democratic Performance? The Interaction of Citizens' Democratic Knowledge and Institutional Level of Democracy", Zeitschrift für Vergleichende Politikwissenschaft 14, núm. 4 (2020), pp. 345-374, https://doi.org/10.1007/s12286-02000467-0

Woldenberg, José, Historia minima de la transición democrática en México, México, El Colegio de México, 2012. 
